\title{
Sexualidade no idoso: percepção de profissionais da geriatria e gerontologia*
}

\author{
Sexuality among the elderly: geriatrics and \\ gerontology professionals' perceptions
}

Dayara Carla Amaral da Costa ${ }^{1}$ Yasmim da Silva Uchôa ${ }^{2}$ Ivan Arnaldo Pamplona da Silva Junior ${ }^{3}$ Saulo de Tarso Saldanha Eremita de Silva ${ }^{4}$ Wiviane Maria Torres de Matos Freitas ${ }^{5}$ Soanne Chyara da Silva Soares ${ }^{6}$

* Recebido em: 12/04/2016. Aprovado em: 17/10/2017.

1 Acadêmica no curso de Bacharelado em Fisioterapia do Centro Universitário do Pará - CESUPA - Belém - Pará - Brasil.

2 Acadêmica no curso de Bacharelado em Fisioterapia do Centro Universitário do Pará - CESUPA - Belém - Pará - Brasil.

3 Acadêmico no curso de Bacharelado em Fisioterapia do Centro Universitário do Pará - CESUPA - Belém - Pará - Brasil.

4 Acadêmico no curso de Bacharelado em Fisioterapia do Centro Universitário do Pará - CESUPA - Belém - Pará - Brasil.

5 Mestre, docente no curso de Bacharelado em Fisioterapia do Centro Universitário do Pará CESUPA - Belém - Pará - Brasil.

6 Centro Universitário de Brasília - UniCEUB Brasília - DF, Brasil.

\section{Resumo}

A pesquisa identificou a percepção dos profissionais da saúde acerca da sexualidade em idosos. Trata-se de estudo quantitativo, observacional, transversal analítico, em instituição especializada na assistência a terceira idade em Belém-PA. Foi aplicado questionário com 20 profissionais da saúde. Os dados foram submetidos à análise estatística com teste $\mathrm{G}$ de aderência. Os profissionais relatam que tiveram formação acadêmica insipiente, que se sentem razoavelmente capacitados para lidar com o tema, que as principais orientações passadas dizem respeito ao uso de preservativos e doenças sexualmente transmissíveis, que as dificuldades de abordar o tema dizem respeito à resistência dos próprios idosos, que a precária abordagem interfere na qualidade de vida dos mesmos. Embora os profissionais reconheçam a importância da sexualidade na integralidade do ser, existe carência na formação profissional, atuação restrita sobre o tema, além de tabus socioculturais que podem interferir na qualidade de vida dos idosos.

Palavras-chave: Sexualidade. Saúde sexual. Profissionais da saúde. Qualidade de vida.

\begin{abstract}
The arm was identify the perception of health professionals about sexuality among the elderly. Quantitative study, observational, analytical cross-sectional in institution specialized on the elderly care in BelémPA. Questionnaire was applied to 20 health professionals. Data were statistically analyzed with $G$ grip test. These professionals related that they had no academic formation regarding aged people sexuality, that they felt reasonably prepared to deal with the issue, that main orientations were about condom utilization and sexually transmitted disease, that the greatest difficulty is their own resistance to talk about the issue, that the professionals recognizes that a poor approach significantly affects aged people quality of life. Although professionals recognize the importance of sexuality in the fullness of being, there is huge lack of professional training, performances restrict, and beyond the socio-cultural taboos that can interfere in the quality of life of the elderly.
\end{abstract}

Keywords: Sexuality. Sexual health. Health professionals. Quality of life. 


\section{Introdução}

O envelhecimento é definido como um processo de progressivas modificações biológicas, psicológicas e sociais ao longo da vida do ser humano. Segundo a Organização Mundial de Saúde, idoso é o indivíduo com idade maior ou igual a 60 anos para os países em desenvolvimento, como é o caso do Brasil (SANTOS; ASSIS, 2011).

De acordo com o Instituto Brasileiro de Geografia e Estatística (2010), nos próximos 20 anos, os idosos representarão quase $13 \%$ da população total, podendo ultrapassar os 30 milhões de pessoas (FRUGOLI; MAGALHÃES, 2011). E, paralelamente a esse crescimento da expectativa de vida, deve evoluir, também, a assistência aos idosos. Dentro desse contexto, os profissionais da saúde necessitam ter conhecimentos científicos das alterações fisiológicas e fisiopatológicas do envelhecimento (SOARES, 2011), favorecendo que os idosos assumam um papel cada vez mais efetivo na sociedade.

Entre as várias vertentes que envolvem a efetividade de uma pessoa na sociedade, inclui-se a sexualidade. De acordo com Labronici e Trentini (2001), a sexualidade envolve uma multidimensionalidade, expressando-se mediante a interação com o outro e manifestando-se nas relações sociais por meio da corporeidade. Contempla, nesse contexto a "maneira de ser e de estar no mundo mediante os Eros que permeiam o cotidiano humano" (LABRONICI; TRENTINI, 2001, p. 68). E, segundo Bernardo; Cortina (2012) o sexo é, apenas, uma das formas de expressar o amor humano por meio das relações afetivas, porém, não é a única forma.

Quando se trata de idosos, Soares et al (2011) relata que há uma carência no questionamento sobre as várias vertentes da sexualidade, inclusive, da vida sexual, pois a maioria dos profissionais de saúde não tem como prática preventiva ou terapêutica, valorizar o tema. Esse fato está relacionado à ínfima formação acadêmico-profissional sobre o tema em idosos, aos constrangimentos devido aos tabus socioculturais existentes, ou mesmo em virtude de os profissionais não perceberem que, mesmo com suas especificidades, atuam, diretamente ou indiretamente, com a autoestima feminina/masculina e suas relações (MASCHIO, 2011; LOCHLAINN; KENNY, 2013).

Assim, muitas vezes, o idoso não é assistido quanto à sua sexualidade, que, de um modo mais sumário, prejudica a prevenção e o tratamento de doenças que têm surgido nessa faixa etária, como a disfunção erétil, dis- paurenia, anorgasmia, entre outros; e, de um modo mais complexo, interfere na qualidade de vida do idoso (SOARES et al, 2011).

Essas concepções negligenciadas decorrentes da redução de sexualidade dos idosos estão evidentes, também, no Caderno de Atenção Básica do Idoso no Ministério da Saúde (2006), sendo um tema pouco explorado diante da vasta diversidade de assuntos contidos, demonstrando a escassa abordagem dos profissionais sobre a temática com o público-alvo, interferindo tanto quanto diabetes, hipertensão, depressão e outras doenças, na qualidade de vida e longevidade dessas pessoas.

Diante desse tema, torna-se essencial identificar a percepção dos profissionais da saúde acerca da sexualidade na terceira idade visto que é um tema pouco discutido e que forma parte integral na personalidade de cada um, necessidade básica e um aspecto que não pode ser separado de outros aspectos da vida.

\section{Metodologia}

$\mathrm{O}$ presente estudo seguiu as normas de pesquisa envolvendo seres humanos (Res. CNS 466/12) do Conselho Nacional de Saúde. Teve início após a aprovação do projeto pelo Comitê de Ética em Pesquisa (CAAE: 19348413.8.000.5169), a autorização da instituição investigada, e a assinatura do Termo de Consentimento Livre e Esclarecido pelos indivíduos envolvidos na pesquisa.

Trata-se de um estudo quantitativo, observacional do tipo transversal analítico, realizada na única instituição pública especializada e exclusiva para assistência ambulatorial à pessoa idosa da região metropolitana de Belém-PA.

A casuística considerada foi de 20 profissionais da saúde de nível superior, em relação ao total de 24 que trabalham no local (4 estavam de férias). Eram de ambos os sexos, pertenciam a todas as faixas etárias, trabalhavam como efetivos da casa especializada em saúde do idoso e aceitaram participar da pesquisa por meio da assinatura do Termo de Consentimento Livre e Esclarecido, no mês de janeiro de 2014.

Para a coleta de dados, foi aplicado questionário por meio de entrevista individualizada para cada participante, a fim de identificar a atuação profissional com os idosos sobre o aspecto da sexualidade. O questionário continha perguntas relacionadas ao perfil do profissional (sexo, idade, formação acadêmica, e tempo de graduação) 
e a questões que relacionavam a sexualidade, formação acadêmica e atuação profissional. A maioria dos dados foi quantificada pelos profissionais em uma escala de 0 a 10 , sendo, posteriormente, atribuída uma qualidade ao número: 0,1 a 3,4 a 6,7 a 9, 10 sendo respectivamente, nada, pouco, mediano, razoável e muito.

Os dados coletados foram tabulados no software Microsoft EXCEL 2010. A análise estatística foi realizada por meio do software BioEstat 5.0. Este estudo foi financiado pelos próprios autores.

\section{Resultados}

O estudo contou com uma casuística de 20 profissionais da área da saúde, sendo 16 do sexo feminino (80\%) e 4 do sexo masculino (20\%). Sendo 6 (30\%) da Medicina, 4 (20\%) da Fisioterapia, 2 (10\%) da Assistência social, 2 (10\%) da Enfermagem, 2 (10\%) da Odontologia, 1 (5\%) da Farmácia, 1 (5\%) da Nutrição, 1 (5\%) da Psicologia e 1 (5\%) da Terapia Ocupacional. A média de idade foi de 41.8 anos ( $D P \pm 12.2$ ). Quanto ao tempo de formação, a média foi de 17.1 anos (12.4). (Tabela 1)

Tabela 1 - Variáveis socioeducacionais

\begin{tabular}{|c|c|c|}
\hline Socioeducacionais & $\mathrm{N}=20$ & Percentual \\
\hline \multicolumn{3}{|l|}{ Sexo } \\
\hline Masculino & 4 & $20.0 \%$ \\
\hline Feminino & 16 & $80.0 \%$ \\
\hline \multicolumn{3}{|l|}{ Idade } \\
\hline Média $\pm D P$ & $41.8 \pm 12.2$ anos & \\
\hline Mediana & 40 anos & \\
\hline Mínimo & 26 anos & \\
\hline Máximo & 73 anos & \\
\hline \multicolumn{3}{|l|}{ Tempo de Formação } \\
\hline Média $\pm D P$ & $17.1 \pm 12.4$ anos & \\
\hline Mediana & 15.5 anos & \\
\hline Mínimo & 2 anos & \\
\hline Máximo & 49 anos & \\
\hline \multicolumn{3}{|l|}{ Profissão } \\
\hline Assistência social & 2 & $10.0 \%$ \\
\hline Enfermagem & 2 & $10.0 \%$ \\
\hline Farmácia & 1 & $5.0 \%$ \\
\hline Fisioterapia & 4 & $20.0 \%$ \\
\hline Medicina & 6 & $30.0 \%$ \\
\hline Nutrição & 1 & $5.0 \%$ \\
\hline Odontologia & 2 & $10.0 \%$ \\
\hline Psicologia & 1 & $5.0 \%$ \\
\hline Terapia ocupacional & 1 & $5.0 \%$ \\
\hline
\end{tabular}

Quanto ao conhecimento profissional sobre a sexualidade (Tabela 2), todos os 20 (100\%) profissionais declaram saber diferenciar sexo de sexualidade. A maioria significativa teve de "nada" (45\%) a "pouco" (30\%) embasamento durante sua formação acadêmica necessária para abordar o tema. Mas, atualmente, a maioria significativa num total de 15 (75\%) profissionais ao menos se sente "razoavelmente" qualificada para tratar da sexualidade com os idosos.

Tabela 2 - Conhecimento profissional acerca da sexualidade em idosos

\begin{tabular}{|c|c|c|c|}
\hline Questionário & $\begin{array}{l}\text { Profissionais } \\
\mathrm{N}=20\end{array}$ & Percentual & p-valor \\
\hline \multicolumn{4}{|c|}{ Sexo versus Sexualidade } \\
\hline Sim & 20 & $100.0 \%$ & \multirow{2}{*}{ NSA } \\
\hline Não & 0 & $0.0 \%$ & \\
\hline \multicolumn{4}{|c|}{ Embasamento na formação acadêmica } \\
\hline Nada & 9 & $45.0 \%$ & \multirow{5}{*}{$\begin{array}{l}0.0250^{*} \\
\text { (teste G) }\end{array}$} \\
\hline Pouco & 6 & $30.0 \%$ & \\
\hline Mediano & 2 & $10.0 \%$ & \\
\hline Razoável & 2 & $10.0 \%$ & \\
\hline Muito & 1 & $5.0 \%$ & \\
\hline \multicolumn{4}{|c|}{ Qualificação atual para atuação } \\
\hline Nada & 1 & $5.0 \%$ & \multirow{5}{*}{$\begin{array}{l}<0.0001^{*} \\
\text { (teste G) }\end{array}$} \\
\hline Pouco & 2 & $10.0 \%$ & \\
\hline Mediano & 1 & $5.0 \%$ & \\
\hline Razoável & 15 & $75.0 \%$ & \\
\hline Muito & 1 & $5.0 \%$ & \\
\hline
\end{tabular}

*Diferença estatisticamente significante $(p<0,05)$. NSA= Não se aplica teste estatístico

Quanto à atuação profissional acerca da sexualidade em idosos (Tabela 3), embora ainda haja profissionais que atribuam de "nada" a "pouco" (35\%) a importância de abordar o tema com os idosos, a maioria (75\%) admite dialogar sobre a sexualidade. E, com dado significativo, metade (50\%) entende que a precária abordagem do tema possa interferir "muito" na qualidade de vida dos idosos. As principais orientações dadas aos idosos foram o "diagnóstico das DST's” e a "importância do uso de preservativos" com 55\% (11) cada. E as maiores dificuldades encontradas em abordar o tema foram a "resistência dos idosos" com 50\% (10), "nenhum" 30\% (6) e o "medo de ser invasivo" $25 \%$ (5). 
Tabela 3 - Atuação profissional acerca da sexualidade em idosos

\begin{tabular}{|c|c|c|c|}
\hline \multirow{2}{*}{ Atuação profissional } & \multicolumn{2}{|c|}{ Profissionais } & \multirow{2}{*}{$p$-valor } \\
\hline & $\mathrm{N}=20$ & Percentual & \\
\hline \multicolumn{4}{|l|}{ Importância na abordagem } \\
\hline Nada & 4 & $20.0 \%$ & \multirow{5}{*}{$\begin{array}{l}0.8419 \\
\text { (teste G) }\end{array}$} \\
\hline Pouco & 3 & $15.0 \%$ & \\
\hline Mediano & 6 & $30.0 \%$ & \\
\hline Razoável & 3 & $15.0 \%$ & \\
\hline Muito & 4 & $20.0 \%$ & \\
\hline \multicolumn{4}{|l|}{ Diálogo sobre sexualidade } \\
\hline Sim & 15 & $75.0 \%$ & \multirow{2}{*}{$\begin{array}{l}0.0222^{*} \\
\text { (teste G) }\end{array}$} \\
\hline Não & 5 & $25.0 \%$ & \\
\hline \multicolumn{4}{|l|}{ Precariedade na abordagem } \\
\hline Nada & 0 & $0.0 \%$ & \multirow{5}{*}{$\begin{array}{l}0.0436^{*} \\
\text { (teste G) }\end{array}$} \\
\hline Pouco & 0 & $0.0 \%$ & \\
\hline Mediano & 3 & $15.0 \%$ & \\
\hline Razoável & 7 & $35.0 \%$ & \\
\hline Muito & 10 & $50.0 \%$ & \\
\hline \multicolumn{4}{|l|}{ Principais orientações ${ }^{1}$} \\
\hline Diagnósticos das disfunções & 9 & $45.0 \%$ & \multirow{6}{*}{ NSA } \\
\hline Diagnósticos das DST's & 11 & $55.0 \%$ & \\
\hline Automedicação & 7 & $35.0 \%$ & \\
\hline Preservativos & 11 & $55.0 \%$ & \\
\hline Outros & 4 & $20.0 \%$ & \\
\hline Nenhum & 0 & $0.0 \%$ & \\
\hline \multicolumn{4}{|c|}{ Dificuldades em abordar 0 assunto ${ }^{1}$} \\
\hline Resistência & 10 & $50.0 \%$ & \multirow{6}{*}{ NSA } \\
\hline Medo & 5 & $25.0 \%$ & \\
\hline Falta de conhecimento & 1 & $5.0 \%$ & \\
\hline Despreparação & 1 & $5.0 \%$ & \\
\hline Outros & 1 & $5.0 \%$ & \\
\hline Nenhum & 6 & $30.0 \%$ & \\
\hline
\end{tabular}

*Diferença estatisticamente significante $(p<0,05)$.

${ }^{1}$ Nesta variável poderia se obter mais de uma resposta. NSA= Não se aplica teste estatístico.

\section{Discussão}

No Brasil, há um aumento percentual de idosos, que somam $11,1 \%$ da população, perfazendo um total de 21 milhões de pessoas. O envelhecimento da população brasileira é reflexo do aumento da expectativa de vida, graças aos avanços tecnocientíficos no campo da saúde que reduz a taxa de mortalidade. As projeções indicam que a população idosa aumentará, continuamente, aproximando-se de $20 \%$ da população total brasileira em 2050 (SCHIMIDT; SILVA, 2013).

Para Berlezi e Van der Sand (2008), as mudanças que vêm ocorrendo em consequência da crescente presença de idosos na sociedade exigem de todos, em especial dos profissionais de saúde, uma nova postura com relação às ações assistenciais a serem desenvolvidas para $\mathrm{o}$ atendimento de suas reais necessidades. E, para assumirem essa nova postura, é necessário que esses profissionais aprofundem seus conhecimentos sobre os hábitos, percepções, costumes, sentimentos, cultura, entre outros atributos dessa população para que possa assisti-la com qualidade.

A sexualidade está presente em todas as dimensões da vida pessoal, profissional, pública ou privada e, ainda, no contexto de saúde ou de doença (MENEZES, 2011). Mesmo assim, a literatura revela que é um tema pouco debatido na formação acadêmica dos profissionais da saúde. Apesar disso, esta pesquisa evidenciou que todos os profissionais entrevistados relatam saber diferenciar sexo de sexualidade. Porém, a maioria significativa destes afirmam que não foram preparados durante suas graduações para abordar o tema com os idosos. Reflexo dessa educação, que não contempla adequadamente esse tema, é que na atualidade $75 \%$ dos profissionais se sentem "razoavelmente" e não "totalmente" preparados para tratar sobre sexualidade com os idosos. Em relação a esse aspecto, não se pode olhar o profissional de saúde e desejar que ele exerça seu papel de modo a contemplar todas as dimensões do ser humano (MENEZES, 2011), uma vez que a sexualidade, sobretudo em idosos, é um tema desafiador desde a formação, como evidenciado na pesquisa. Identifica-se, então, que é iminente a necessidade de uma educação gerontológica nos projetos pedagógicos de todo curso da saúde, de uma inovação curricular nesse momento histórico que suscita inúmeras questões sobre o envelhecimento e a velhice (ARCOVERDE, 2006).

Mesmo com deficit no embasamento teórico, 75\% dos profissionais entrevistados relataram dialogar com os idosos sobre sexualidade. No entanto, 35\% relataram que a abordagem do tema com os idosos tem de "nada" a "nenhuma" importância, demonstrando que a sexualidade em idosos, ainda, não é prioridade para os profissionais (BASTOS, 2012; EAST; HUTCHINSON, 2013). Mudanças socioculturais em atitudes relativas à sexualidade e ao envelhecimento vêm desafiando o estereótipo tradicional da "velhice assexuada", na medida em que a função sexual passou a ser vista como um componente vital para se alcançar um envelhecimento de sucesso em geral (CEZAR; AIRES; PAZ, 2012). Dentro desse contexto, a percepção 
de que abordagem precária do tema com os idosos pode influenciar na qualidade de vida dos idosos é relatada por $50 \%$ dos profissionais envolvidos na pesquisa. A realidade é que o envelhecimento representa um processo biopsicossociocultural e, por essa natureza, gera demandas complexas e exige cuidado diferenciado (CEZAR; AIRES; PAZ, 2012).

Devido à educação repressora que muitos idosos receberam no passado, esse tema não é facilmente trabalhado na relação terapeuta-paciente, já que os idosos não se sentem bem em expressar opiniões sobre o assunto (SOARES, 2011). Corroborando esse aspecto, a presente pesquisa destaca que a principal dificuldade encontrada por metade dos profissionais em abordar o tema é a "resistência dos idosos" em discutir sobre o assunto, seguido do "medo do profissional de ser invasivo". Barreiras como esta, além da barreira física, são apontadas por Dominguez e Barbagallo (2016) como sendo consequência do estereótipo social que influencia a formação e atuação dos profissionais da saúde. Para Cezar, Aires e Paz (2012), é obvio que ações e reações surgem nos idosos, pois são pessoas que vieram de uma época em que nem se cogitava falar sobre o assunto e estas, muitas vezes, não são bem compreendidas pelos profissionais de saúde devido ao desconhecimento sobre todos os aspectos que envolvem o cuidado ao paciente idoso. Assim, torna-se necessário o conhecimento a cerca de como eles percebem e vivenciam sua sexualidade, o que permitirá a construção de conhecimento sobre o assunto, acabando com os mitos, tabus e informações errôneas.

Quando se tratam de orientações que, sumariamente, dizem respeito à sexualidade, a pesquisa revelou que a atuação profissional em relação aos idosos está direcionada ao diagnóstico das Doenças Sexualmente Transmissíveis (DST) e à importância do uso de preservativos. Mesmo se orientações dessa natureza são dadas, no Brasil, observa-se a crescente porcentagem, de 7\% em 1996 para 13\% em 2004, de idosos infectados por DST. Esse aumento se deve às incipientes campanhas de saúde acerca da sexualidade para esses cidadãos. Medidas preventivas efetivas, especialmente no que se refere ao uso de preservativos, são, ainda, incipientes entre os idosos que nos outros segmentos populacionais. Assim, prevenção às DST nessa faixa etária acaba se tornando um desafio para a política pública (NERY; VALENÇA, 2014).

Os dados dessa pesquisa, no que concerne à orientação do idoso demonstram uma lacuna no pensar e agir dos profissionais de saúde quando o assunto é a sexualidade das pessoas idosas. Buscar conhecer a sexualidade do paciente idoso não se refere apenas em identificar se possui ou não alguma DST. Há evidências que enfermeiros mais capacitados no aspecto da sexualidade atuam com clareza, abertura e honestidade, quebrando tabus socioculturais e interferindo positivamente na saúde sexual de idosos (FARRELL; BELZA, 2012; RONEY; KAZER, 2015). Percebe-se, então, que a promoção da saúde do idoso aponta para a necessidade de um processo de capacitação de profissionais visando à melhoria das condições de vida e de saúde na terceira idade (BERNARDO; CORTINA, 2012).

\section{Conclusão}

Conclui-se que, embora os profissionais da saúde reconheçam seu papel na saúde dos idosos, eles não se consideram devidamente capacitados para discutir assuntos referentes à sexualidade com essa população. Fato este que tem relação com déficit desde a formação acadêmica que perdura, em menor escala, até a atuação profissional. Nota-se, ainda, que esse profissional, inserido num contexto sociocultural em que existem tabus relacionados à sexualidade na terceira idade, sente-se às vezes constrangido para discutir esse tema com os idosos já que há resistência advinda dessa população. Além disso, os profissionais reconhecem que sua atuação no aspecto da sexualidade influencia positivamente na qualidade de vida dos idosos. Essa pesquisa abre precedentes para estudos mais direcionados à formação e atuação do profissional de saúde, delimitando as possíveis fragilidades e potencialidades para uma atenção integral e igualitária do ser humano em todas as fases da vida e em todos os aspectos da vida, destacando-se neste trabalho, os idosos e a sexualidade.

\section{Referências}

ARCOVERDE, M. A. M. A percepção da sexualidade do corpo idoso. 2006. 96 f. Dissertação (Mestrado) Programa de Pós graduação em Enfermagem - Setor de Ciências da Saúde, Universidade Federal do Paraná, Curitiba, 2006.

BASTOS, C. C. Importância atribuída ao sexo por idosos do município de Porto Alegre e associação com a autopercepção de saúde e o sentimento de felicidade. Revista Brasileira de Geriatria e Gerontologia, Rio de Janeiro, v. 15, n. 1, p. 87-95, jan./mar. 2012. 
BERLEZI, J. J.; VAN DER SAND, I. C. P. Concepção de professores e estudantes de enfermagem acerca da sexualidade: um estudo qualitativo. Revista Contexto e Saúde, Ijuí, v. 8, n. 15, p. 45-53, jul./dez. 2008.

BERNARDO, R.; CORTINA, I. Sexualidade na terceira idade. Revista Enfermagem UNISA, Santo Amaro, v. 13, n. 1, p. 74-78, 2012.

BRASIL. Ministério da Saúde. Secretaria de Atenção à Saúde. Departamento de Atenção Básica. Envelhecimento e saúde da pessoa idosa. Brasília: Ministério da Saúde, 2006.

CEZAR, A. K.; AIRES, M.; PAZ, A. P. Prevenção de doenças sexualmente transmissíveis na visão de idosos de uma estratégia de Saúde da Família. Revista Brasileira de Enfermagem, Brasília, v. 65, n. 5, p. 745-750, set/ out. 2012.

DOMinguEZ, L. J.; BARBAGALLO, M. Ageing and sexuality. European Geriatric Medicine, Paris, v. 7, n. 6, p. 512-518, Dec. 2016.

EAST, L.; HUTCHINSON, M. Moving beyond the therapeutic relationship: a selective review of intimacy in the sexual health encounter in nursing pratice. Journal of Clinical Nursing, Oxford, v. 22, n. 23-24, p. 3568-3576, Dec. 2013.

FARRELL, J.; BELZA, B. Are older patients comfortable discussing sexual health with nurses? Nursing research, New York, v. 61, n. 1, p. 51-57, jan./feb. 2012.

FRUGOLI, A.; MAGALHÃES-JUNIOR, C. A. O. A sexualidade na terceira idade na percepção de um grupo de idosas e indicações para a educação sexual. Arquivos de Ciências da Saúde UNIPAR, Umuarama, v. 15, n. 1, p. 85-93, jan./abr. 2011.

INSTITUTO BRASILEIRO DE GEOGRAFIA E ESTATÍSTICA - IBGE. Atlas do censo demográfico 2010. Rio de Janeiro: IBGE, 2013.

LABRONICI, L. M.; TRENTINI, M. Eros proporcionando a compreensão da sexualidade das enfermeiras. Revista Cogitare Enfermagem, Curitiba, v. 6, n. 1, p. 67-74, jan./jun. 2001.

LOCHLAINN, M. N.; KENNY, R. A. Sexual activity and aging. Journal of the American Medical Directors Association, Hagerstown, v. 14, n. 8, p. 565-572, Aug. 2013.

MASCHIO, M. B. M. et al. Sexualidade na terceira idade: medidas de prevenção para doenças sexualmente transmissíveis e a AIDS. Revista Gaúcha de Enfermagem, Porto Alegre, v. 32, n. 3, p. 583-589, set. 2011.
MENEZES, I. C. D. Percepção da sexualidade na terceira idade: uma oportunidade para abordagem do tema. 2011. 67 f. Trabalho de Conclusão de Curso. (Graduação em Enfermagem) - Universidade Estadual da Paraíba, Campina Grande, 2011.

NERY, V. A. S.; VALENÇA, T. D. C. Sexo e sexualidade no processo de envelhecimento. Revista Eletrônica da Fainor, Vitória da Conquista, v. 7, n. 2, p. 20-32, jul./dez. 2014.

RONEY, L.; KAZER, M. W. Geriatric sexual experiences: the seniors tell all. Applied Nursing Research, Philadelphia, v. 28, n. 3, p. 254-256, Aug. 2015.

SANTOS, A. F. M.; ASSIS, M. Vulnerabilidade das idosas ao hiv/aids: despertar das políticas públicas e profissionais de saúde no contexto da atenção integral: revisão de literatura. Revista Brasileira de Geriatria e Gerontologia, Rio de Janeiro, v. 14, n. 1, p. 147-157, jan./mar. 2011.

SCHIMIDT, T. C. G.; SILVA, M. J. P. Influência das características físicas humanas na comunicação do profissional da saúde com o idoso. Revista Mineira de Enfermagem, Belo Horizonte, v. 17, n. 3, p. 510-516, jul./set. 2013.

SOARES, A. M. et al. AIDS no idoso. In: FREITAS, E. V. et al. Tratado de geriatria e gerontologia. 3. ed. Rio de Janeiro: Guanabara Koogan, 2011. p. 918-929.

\section{Agradecimentos}

À Secretaria Municipal de Saúde e Meio Ambiente de Belém (PA), em nome da Casa do Idoso e seus profissionais e usuários que permitiram e contribuíram para a realização desta pesquisa.

FINANCIAMENTO DA PESQUISA: A pesquisa foi financiada pelos próprios autores, sem financiamento externo. 